\title{
Nuevas Drogas Psicoactivas
}

\section{New Psychoactive Drugs}

\author{
Helen Dolengevich-Segal*, Beatriz Rodríguez Salgado**, Jorge Gómez-Arnau Ramírez*, Daniel \\ SÁNCHEZ-MATEOS***
}

*Servicio de Psiquiatría. Hospital Universitario del Henares. Coslada (Madrid). **Hospital Ramón y Cajal. Centro de Salud Mental de San Blas, Madrid. ***Hospital La Fe, Valencia.

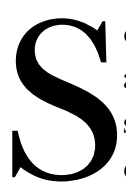

e denominan Nuevas Drogas Psicoactivas (NDP) a aquellas que no están reguladas por la Convención sobre Drogas Psicotrópicas de las Naciones Unidas de 1971 (United Nations Office on Drugs and Crime, 2013). Se trata de moléculas no fiscalizadas que pretenden emular los efectos psicoactivos de las drogas ilegales. En el concepto caben, asimismo, no sólo sustancias de nueva síntesis, sino también otras ya conocidas que se consumen fuera de los contextos tradicionales, así como nuevas vías de distribución para las mismas. Es en este sentido en el que internet desempeña un papel fundamental, pues a través de la red es relativamente sencilla la obtención de drogas conocidas como "euforizantes legales" o legal highs, así como "productos químicos de investigación” o research chemicals. Se venden como "incienso místico", "limpiadores de pipas de agua", "suplementos alimenticios", "sales de baño", "artículos de coleccionista” o "abono para plantas", con la advertencia de que no son aptas para el consumo humano. No obstante, es posible encontrar numerosas páginas web en las que se detallan dosis, formas de consumo, combinaciones, complicaciones posibles y efectos esperados. La naturaleza de estas sustancias es variada: desde productos herbales, en los que se aprovecha la falsa idea de que por ser naturales son inocuos, a sustancias químicas, sintéticas y semisintéticas, farmacéuticas (intermediarios químicos utilizados para la preparación de otras sustancias) o mezclas de los anteriores. Con frecuencia no se detalla la composición exacta de los productos, en los que la adulteración también es habitual. La actividad psicoactiva también es diversa: estimulantes, sedantes, empatógenos, alucinógenos, analgésicos. De hecho, con la moderna composición de sustancias, existen algunas que en sí mismas pueden suponer un "policonsumo", al tener la capacidad de incidir en diversos receptores neuronales y presentar diferentes efectos. Tal es el caso del cannabinoide sintético STS-35, que actúa como ligando de los receptores cannabinoides, como antagonista glutamatérgico del receptor tipo NMDA (la misma acción que lleva a cabo el MK-801, molécula utilizada para la inducción en animales de psicosis experimentales) y como agonista serotoninérgico; o del opioide sintético MT-45, que cuenta con una estructura piperazínica, de la que se espera un efecto estimulante, a la par que actúa sobre receptores $\mu$ opioides (Siddiqi, Verney, Dargan y Wood, 2015). Fuera de las redes comerciales habituales, existe una Deep web, en la que la información encriptada sortea la vigilancia de las autoridades, lo que permite el tráfico de productos ilegales. En los últimos años han sido clausurados diversos portales en los que se venden drogas ilícitas clásicas, como cocaína, heroína, MDMA o anfetaminas.

El último informe del Observatorio Europeo sobre Drogas y Toxicomanías basado en los datos recolectados a través del Sistema de Alerta Temprana de la Unión Europea señala que en el año 2014 fueron detectadas 101 nuevas sustancias psicoactivas de un total de 450 identificadas por

Recibido: Mayo 2015; Aceptado: Mayo 2015

Enviar correspondencia a:

Helen Dolengevich Segal. Servicio de Psiquiatría. Hospital Universitario del Henares. Avda. de Marie Curie s/n, 28822, Coslada (Madrid). E-mail: e.dolengevich@gmail.com 
el sistema desde sus inicios, en 1997. De entre ellas, destacan por su frecuencia los cannabinoides sintéticos y las catinonas sintéticas (European Monitoring Centre for Drugs and Drug Addiction, 2015). La literatura científica reciente también se hace eco de la llegada a Europa de nuevas drogas, tanto a través de análisis toxicológicos como de reportes de casos de complicaciones clínicas vinculadas al consumo (Wood, Sedefov, Cunningham y Dargan, 2015).

La aparición de trastornos psicopatológicos entre la población que consume NDP nos invita a la reflexión acerca de la posible presencia de una patología dual, la cual se refiere no sólo a la comorbilidad entre un diagnóstico psiquiátrico y un trastorno por consumo de sustancias, sino a la existencia de una vulnerabilidad común. El término patología dual alude a fenotipos psicopatológicos en los que se postula una asociación en la que caben condicionantes genéticos y ambientales. En la confluencia de sendas patologías ha sido señalada la posible existencia de un sustrato neuronal común, bajo el que se alojan respuestas a diferentes tipos de estrés, así como las hipótesis de la automedicación y de la autorregulación o la influencia de factores ambientales y del estilo de vida (Szerman et al., 2013; Volkow, 2001).

En este sentido, se actualiza la definición de psiconauta, acuñada por Jünger (1952), al referirse a quienes utilizan enteógenos para explorar su psique. Ha sido propuesto el término e-psychonaut en alusión a la importancia de la red para la obtención tanto de material psicoactivo como de información acerca de los efectos, la farmacología, los riesgos o las combinaciones posibles (Davey, Schifano, Corazza, Deluca y Psychonaut Web Mapping Group, 2012). Epidemiológicamente, los psiconautas suelen ser varones, entre la tercera y cuarta década de la vida, con un nivel educativo alto y una marcada inclinación al policonsumo. En los últimos años también se añaden adolescentes tardíos y adultos jóvenes de ambos sexos, todos ellos con escasa experiencia (Delegación del Gobierno para el Plan Nacional sobre Drogas, 2015). En el caso del consumo de NDP y la presencia de psicopatología, podría hablarse de psiconauta dual en referencia tanto a los sujetos con una posible enfermedad mental que buscan la administración específica de estas sustancias, como a aquellas personas en los que se despiertan síntomas psicopatológicos mantenidos tras su consumo, poniendo en evidencia una vulnerabilidad subyacente (Dolengevich-Segal, Rodríguez-Salgado, Gómez-Arnau, Rabito-Alcón y Correas-Lauffer, 2014).

Los datos orientan sobre la importancia del fenómeno, ya que supone un cambio tanto en las sustancias mismas como en los patrones de su consumo e incluso en el perfil epidemiológico de los consumidores, todo lo cual conlleva la necesidad de actualizar los conocimientos sobre el abordaje de las posibles complicaciones médicas y psicopatológicas (Kersten y McLaughlin, 2014).

\section{Referencias}

Davey, Z., Schifano, F., Corazza, O., Deluca, P. y Psychonaut Web Mapping Group. (2012). e-Psychonauts: conducting research in online drug forum communities. Journal of Mental Health, 21, 386-94.

Delegación del Gobierno para el Plan Nacional sobre Drogas (2015). Encuesta sobre alcohol y drogas en la población general en España (EDADES 2013/2014). Ministerio de Sanidad, Servicios Sociales e Igualdad.

Dolengevich-Segal, H., Rodríguez-Salgado, B., GómezArnau, J., Rabito-Alcón, M. F. y Correas-Lauffer, J. (2014). Psychoactive substance use in the Internet era. Revista de Patología Dual, 1, 3-9.

European Monitoring Centre for Drugs and Drug Addiction (2015). New psychoactive substances in Europe. An update from the EU Early Warning System. Publications Office of the European Union, Luxembourg.

Jünger, E. (1987). Visita a Godenholm. Madrid: Alianza editorial (trabajo original publicado en 1952).

Kersten, B. P. y McLaughlin, M.E. (2015). Toxicology and management of novel psychoactive drugs. Journal of Pharmacy Practice, 28, 50-65. doi: 10.1177/0897190014544 814.

Siddiqi, S., Verney, C., Dargan, P. y Wood, D. M. (2015). Understanding the availability, prevalence of use, desired effects, acute toxicity and dependence potential of the novel opioid MT-45. Clinical Toxicology (Philadelphia), 53, 54-59. doi: 10.3109/15563650.2014.983239.

Szerman, N., Martínez-Raga, J., Peris, L., Roncero, C., Basurte, I., Vega, P. y Casas, M. (2013). Rethinking Dual Disorders/Pathology. AddictiveDisorders E TheirTreatment, 12, 1-10. doi: 10.1097/ADT.0b013e31826e7b6a.

United Nations Office on Drugs and Crime (2013). The Challenge of New Psychoactive Substances. Disponible en: http://www.unodc.org/documents/scientific/NPS _2013_SMART.pdf

Volkow, N. (2001). Drug abuse and mental illness: progress in understanding comorbidity. American Journal of Psychiatry, 158, 1181-1183.

Wood, D. M., Sedefov, R., Cunningham, A., Dargan, P. I. (2015).Prevalence of useandacute toxicityassociated with the use of NBOMe drugs. Clinical Toxicology (Philadelphia), 53, 85-92. doi: 10.3109/15563650.2015.1004179. 